\title{
TU/e ENHOUEN

\section{Mixed-mode and chaotic oscillations in a simple model of an electrochemical oscillator}

\section{Citation for published version (APA):}

Koper, M. T. M., \& Gaspard, P. (1991). Mixed-mode and chaotic oscillations in a simple model of an electrochemical oscillator. Journal of Physical Chemistry, 95(13), 4945-4947.

https://doi.org/10.1021/j100166a009

DOI:

10.1021/j100166a009

Document status and date:

Published: 01/01/1991

\section{Document Version:}

Publisher's PDF, also known as Version of Record (includes final page, issue and volume numbers)

\section{Please check the document version of this publication:}

- A submitted manuscript is the version of the article upon submission and before peer-review. There can be important differences between the submitted version and the official published version of record. People interested in the research are advised to contact the author for the final version of the publication, or visit the $\mathrm{DOI}$ to the publisher's website.

- The final author version and the galley proof are versions of the publication after peer review.

- The final published version features the final layout of the paper including the volume, issue and page numbers.

Link to publication

\section{General rights}

Copyright and moral rights for the publications made accessible in the public portal are retained by the authors and/or other copyright owners and it is a condition of accessing publications that users recognise and abide by the legal requirements associated with these rights.

- Users may download and print one copy of any publication from the public portal for the purpose of private study or research.

- You may not further distribute the material or use it for any profit-making activity or commercial gain

- You may freely distribute the URL identifying the publication in the public portal.

If the publication is distributed under the terms of Article 25fa of the Dutch Copyright Act, indicated by the "Taverne" license above, please follow below link for the End User Agreement:

www.tue.nl/taverne

Take down policy

If you believe that this document breaches copyright please contact us at:

openaccess@tue.nl

providing details and we will investigate your claim. 


\title{
Mixed-Mode and Chaotic Oscillations In a Simple Model of an Electrochemical Oscillator
}

\author{
Marc T. M. Koper*, ${ }^{\star \dagger}$ and Pierre Gaspard ${ }^{\ddagger}$ \\ Service de Chimie Physique, Université Libre de Bruxelles, Campus Plaine C.P.231, Boulevard du Triomphe, \\ B-1050 Brussels, Belgium (Received: April 3, 1991)
}

\begin{abstract}
We present some preliminary results obtained with a simple three-variable model that should adequately describe the mixed-mode and chaotic oscillations observed during the reduction of indium(III) at mercury in the presence of thiocyanate. This model is the first to satisfactorily reproduce such complex dynamic behavior in an electrochemical system.
\end{abstract}

In the past couple of years, electrochemical reactions have proven to be excellent paradigms in the study of nonlinear dynamics in chemical kinetics. Such diverse systems as the electrodissolution of copper in an acidic environment, ${ }^{1,2}$ the oxidation of formaldehyde or formic acid at platinum, ${ }^{3}$ the reduction of hydrogen peroxide ${ }^{4}$ or the oxidation of hydrogen ${ }^{5}$ at platinum, and the reduction of indium(III) at mercury in the presence of thiocyanate ${ }^{6}$ have been shown to exhibit typical far-from-equilibrium behavior like multistability, oscillations (periodic, quasiperiodic, multiperiodic, often of the mixed-mode type), and chaos. So far, however, there does not exist, for any one of these systems, a complete and reliable theory or model that helps us in understanding why electrochemical reactions are so easily persuaded to display a dynamic behavior whose embedding phase space should at least be three-dimensional.

Of concern in this Letter are some preliminary results obtained with a three-variable model that seems to be, to the best of our knowledge, the first physically reasonable model that is able to explain mixed-mode and chaotic oscillations in an electrochemical system. Our model is in principle concerned with describing the spontaneous current oscillations observed during the reduction of indium(III) catalyzed by thiocyanate at a hanging mercury drop electrode, but we believe that the model possesses some general features that could make it promising in the interpretation of complex oscillations in other, more intricate systems as well.

The system is described by diffusion of In(III) in an electrolyte solution containing a large excess of sodium thiocyanate, coupled with a heterogeneous reaction at the hanging mercury drop. The general equations of the system are the following

$$
\begin{gathered}
C_{\mathrm{d}} \frac{\mathrm{d} E}{\mathrm{~d} t}=\frac{V-E}{R_{\mathrm{s}}}-A n F k_{\mathrm{f}}(E) C_{r=a}(t) \\
\frac{\partial C}{\partial t}=D\left(\frac{\partial^{2} C}{\partial r^{2}}+\frac{2}{r} \frac{\partial C}{\partial r}\right) \\
D\left(\frac{\partial C}{\partial r}\right)_{r=a}(t)=k_{\mathrm{f}}(E) C_{r=a}(t), \quad C_{r=\infty}=C_{\text {bulk }}
\end{gathered}
$$

Equation 1 describes the electric charge conservation at the electrode. ${ }^{7} \quad V$ is the adjustable potential difference acting on the entire circuit, $E$ is the electrode potential, $C_{\mathrm{d}}$ is the capacity of the electrical double layer, $R_{\mathrm{s}}$ is the external series resistance, $A$ is the available drop area, $n$ is the number of electrons involved in the charge-transfer reaction, $F$ is the Faraday constant, and $k_{\mathrm{f}}(E)$ is the overall rate constant for the indium(III) reduction in the presence of thiocyanate. We shall define here below this rate as a function of the electrode potential $E$. Equation 2 is the diffusion equation for the indium(III) concentration $C$ in the spherical geometry of the drop. Equations 3 give the boundary conditions for (2) at the surface of the drop of radius $a$ and at a large distance in the bulk.

\footnotetext{
* To whom correspondence should be addressed.

+ Boursier de la Communaute française de Belgique. Permanent address: Vakgroep Electrochemie, Rijksuniversiteit Utrecht, Utrecht, The Netherlands

"Chercheur Qualifie" of the National Fund for Scientific Research (Belgium).
}

To capture the essence of the phenomenon of mixed-mode oscillations, we shall simplify (1)-(3) in the following way. The electrolyte around the drop of radius $a$ is divided in two fictive spherical diffusion layers which are both of thickness $a / 2$. We use here an extension of the well-known Cottrell result ${ }^{8}$ that the diffusion layer thickness can be estimated by the drop radius. Furthermore, we assume that the indium concentration is uniform at the value $C_{\text {bulk }} u$ in the layer neighboring the electrode while the concentration has the uniform value $C_{\text {bulk }} w$ in the nextneighboring layer. Outside these two layers, i.e., outside a fictive sphere of radius $2 a$, we assume that the concentration has reached the bulk value, $C_{\text {bulk. }}$. These assumptions allow us to reduce eqs $1-3$ to the following three-dimensional vector field

$$
\begin{gathered}
\frac{\mathrm{d} e}{\mathrm{~d} t}=\frac{v-e}{r}-m k(e) u \\
\frac{\mathrm{d} u}{\mathrm{~d} t}=-k(e) u+d(w-u) \\
\frac{\mathrm{d} w}{\mathrm{~d} t}=\frac{19}{333} d(16-25 w+9 u)
\end{gathered}
$$

In eqs $4 e$ is the dimensionless electrode potential, $v$ is the circuit potential, $r$ is the adjustable series resistance (Ohmic solution resistance included), $m$ is some dimensionless parameter proportional to the bulk concentration of the indium(III), $d$ is a parameter proportional to its diffusion coefficient, $u$ and $w$ are its normalized concentrations, and $k(e)$ is the dimensionless heterogeneous rate constant. The electric current $i$ flowing through the circuit is equal to $(v-e) / r$.

While the first equation in (4) is an expression of the conservation of current, the second equation is a mass balance equation for the surface concentration $u$, taking into account the chemical conversion at the interface and the diffusion toward it from the next-neighboring layer. The coefficients in the evolution equation for $w$ stem from the spherical geometry and from our assumption about the thickness of the different diffusion layers. The choice of this geometry is to some extent arbitrary but does not change qualitatively the following results. In particular, we observed the same behaviors replacing these coefficients by those of second spatial difference.

The dimensionless rate constant $k(e)$ for the reduction of $\operatorname{In}($ III) in the presence of thiocyanate can be written as ${ }^{7}$

$$
k(e)=k_{1} \theta^{2}+k_{2} \exp \left(n \alpha\left(e-e^{0}\right)\right)
$$

The first term is the translation of the catalyzing action of the

(1) Albahadily, F. N.; Ringland, J.; Schell, M. J. Chem. Phys. 1989, 30 813. Schell, M.; Albahadily, F. N. J. Chem. Phys. 1989, 90, 822.

(2) Bassett, M. R.; Hudson, J. L. J. Phys. Chem. 1988, 92, 6963. Bassett, M. R.; Hudson, J. L. J. Phys. Chem. 1989, 93, 2731.

(3) Schell, M.; Albahadily, F. N.: Safar, J.; Xu, Y. J. Phys. Chem. 1989. 93, 4806. Xu, Y.; Schell, M. J. Phys. Chem. 1990, 94, 7137.

(4) Fetner, N.; Hudson, J. L. J. Phys. Chem. 1990, 94, 6506.

(5) Krischer, K.; Lübke, M.; Wolf, W.; Eiswirth, M.; Ertl, G. Chaos and Interior Crisis in an Electrochemical Reaction, preprint. Krischer, K. Dissertation, Freien Universität Berlin, 1990.

(6) Koper, M. T. M.; Sluyters, J. H. J. Electroanal. Chem. 1991, 303, 65.

(7) Koper, M. T. M.; Sluyters, J. H. J. Electroanal. Chem. 1991, 303, 73

(8) Bard, A. J.; Faulkner. L. R. Electrochemical Methods, Fundamentals and Applications; Wiley: New York, 1980. 

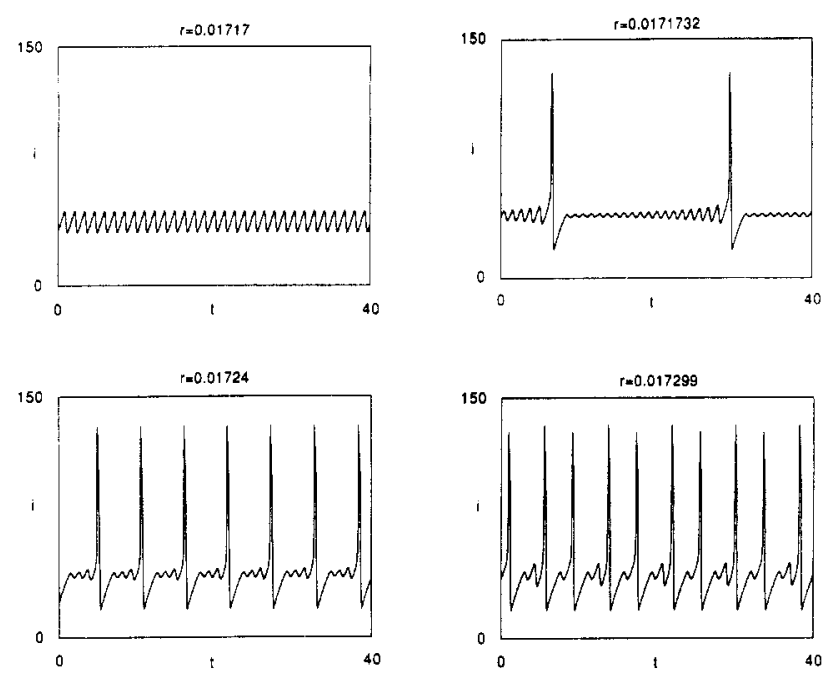

Figure 1. Typical oscillatory time series for the current $i$ obtained for eqs 4-6. Depicted are, for the indicated values of $r$, a small-amplitude oscillation, a MMO state $1^{19}$, a MMO state $1^{3}$, and a typical "Farey" state $1^{1} 1^{2}$. Note the small $r$ domain in which these transitions take place. All other parameter values were fixed at $v=37, d=1, m=120, k_{1}=$ 2.5, $k_{2}=0.01, e_{\mathrm{d}}=35, b=0.5, n \alpha=0.5$, and $e^{0}=30$.

thiocyanate that is specifically adsorbed at the mercury electrode and is in accordance with the kinetic mechanism proposed by Pospisil and de Levie. ${ }^{9}$ The second term (with $\alpha$ the electrontransfer coefficient and $e^{0}$ the dimensionless standard potential) expresses the much slower, uncatalyzed indium reduction. Owing to the interplay of the specific adsorption and Coulombic repulsion between the (negatively charged) thiocyanate $\mathrm{SCN}^{-}$and the electrode surface, the relative thiocyanate occupancy $\theta$ will follow a sigmoidal function of $e$, which we have expressed with ${ }^{7}$

$$
\theta= \begin{cases}1 & \text { for } e \leq e_{\mathrm{d}} \\ \exp \left(-b\left(e-e_{\mathrm{d}}\right)^{2}\right) & \text { for } e>e_{\mathrm{d}}\end{cases}
$$

Note that the desorption of the $\mathrm{SCN}^{-}$with increasing cathodic polarization (i.e., the potential $e$ ) results in a negative $\mathrm{d} k(e) / \mathrm{d} e$ in some potential interval. In general terms, this behavior can be interpreted as an effective negative impedance that is unstabilizing the system and as such announcing the possibility of oscillations. ${ }^{10}$

The model (4) is an extension of a two-variable model earlier proposed by one of us. ${ }^{7}$ The crucial difference between our earlier two-dimensional model and eqs 4 is the way in which we describe the diffusion from the bulk toward the electrode/electrolyte interface. Instead of supposing that the flux at the interface is proportional to $(1-u)$, i.e., the difference in bulk and surface concentration, we have introduced here a third variable, the concentration $w$ halfway in the diffusion layer, that accounts for the fact that, in reality, the concentration profile may be curved when $u$ is changing. Both the previous and the present model appear as truncated versions of the full problem, governed by a partial differential equation. Although our approach can seem a caricature of reality, we will show that the model eqs 4 already contain the minimal ingredients necessary to describe complex behaviors like mixed-mode oscillations and chaos in an electrochemical system.

We carried out a one-parameter analysis of the flow defined by eqs $4-6$ to illustrate the main aspects of the system. We have fixed all parameter values, apart from the resistance $r$, whose value we will vary. The fixed parameter values can be found in the caption of Figure 1. For this set of parameters, the flow admits only one stationary state, regardless of the value of $r$.

Hopf Bifurcation from the Low-Current State. Initially, at low $r$, this fixed point is a stable node at small current and high electrode potential. It is changed into a stable focus at $r=$

(9) Pospisil, L, de Levie, R. J. Electroanal. Chem. 1970, 25, 245.

(10) de Levie, R. J. Electroanal. Chem. 1970, 25, 257.

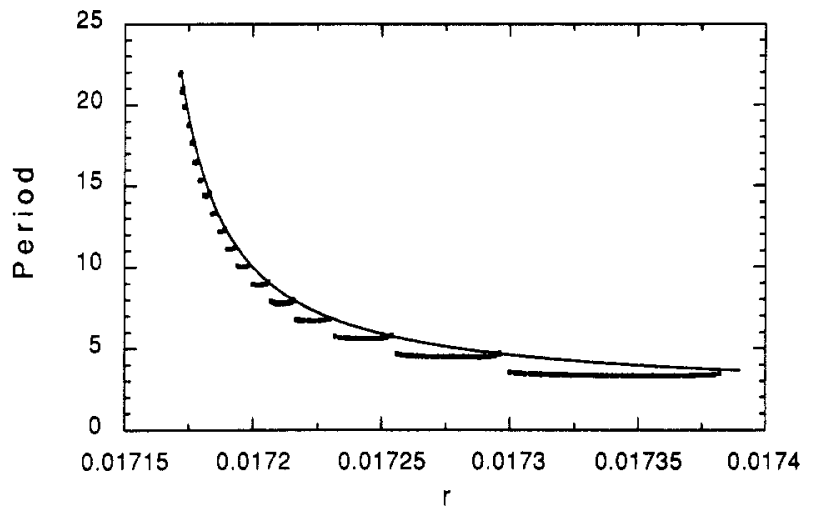

Figure 2. Diagram of the period of the MMO versus the control parameter $r$. The dots are the results of numerical integration of the model eqs 4-6, while the line is the theoretical prediction based on the formula (8) with the parameters $T_{0}=2.1, r_{\mathrm{c}}=0.0171722$, and $c=0.16$.

0.015763 , which in turn undergoes a Hopf bifurcation at $r=$ 0.017155

Mixed-Mode Oscillations (MMO). Shortly hereafter, still raising $r$, the small-amplitude oscillation is destroyed when a sudden relaxation spike appears on the scene, creating the first of a sequence of mixed-mode oscillatory (MMO) states. Some of these states are depicted in Figure 1, and we shall indicate them with a symbolic notation $L^{S}, L$ giving the number of large and $S$ the number of small oscillations in one period. Similar states are also observed at the low-resistance boundary of the oscillatory region in the potential-resistance parameter plane of the In/SCN oscillator. ${ }^{6}$ With increasing $r$, we observe the sequence

$$
1^{21}, 1^{20}, 1^{19}, \ldots, 1^{3}, 1^{3}\left(1^{2}\right)^{n}, 1^{2}, 1^{2}\left(1^{1}\right)^{n}, 1^{1}, 1^{1}\left(1^{0}\right)^{n}, 1^{0}
$$

with $n$ going from 1 to 8 or 10 in our numerical computation. In the right-hand part of the sequence, two neighboring states show interjacent periodic states that are concatenations of the two principal states. In the left-hand part, the windows of stability of these interjacent periodic states are so small that we do not observe them. The bifurcation sequence (7) thus presents two complementary regimes whenever $r$ is low or higher.

Incomplete Homoclinic Scenario. At low $r$ in the sequence, the stability eigenvalues $(\rho \pm i \omega, \lambda)$ of the saddle focus satisfy $|\rho / \lambda|$ $<1$, which is one of Shil'nikov's necessary conditions to have dynamical chaos. ${ }^{11}$ However, the reinjection flow is so contractive that the chaos is squeezed away and there remains only the typical bifurcation sequence of MMO. The role of the saddle focus is demonstrated by the fact that the diagram of the period of MMO versus the control parameter $r$ is reproduced by the formula

$$
T(r)=T_{0}+\rho(r)^{-1} \ln \left[\left(r-r_{\mathrm{c}}\right)^{2}+c^{2}\right]^{1 / 2}
$$

where $\rho(r)$ is the real part of the unstable eigenvalue of the saddle focus which is changing with $r$ while $r_{\mathrm{c}}$ is the critical parameter value for the end of the sequence (see Figure 2). The incompleteness of the homoclinic scenario appears in the constant $c$, which expresses the fact that exact reinjection on the saddle focus creating a complete homoclinic connection does not occur on account of the strong contractivity of the flow. ${ }^{12}$

Incomplete Farey Sequence. The sequence (7) is very reminiscent of the incomplete Farey sequence observed by Schell and co-workers in the electrodissolution of copper in phosphoric acid. ${ }^{1}$ An incomplete Farey sequence like the one described here should not be confused with the complete, unpruned one that is known to be created at the critical surface of mappings of the circle onto itself. ${ }^{13}$ Rather, they are associated with two extremum maps modeling reinjecting phase space flows, ${ }^{14}$ like they are known to typically occur near homoclinic tangencies. ${ }^{14-16}$

(11) Shil'nikov, L. P. Sov. Math. Dokl. 1965, 6, 163; Math. USSR Sb. $1970,10,91$.

(12) Gaspard, P.; Wang, X.-J. J. Stat. Phys. 1987, 48, 151

(13) Jensen, M. H.; Bak, P.; Bohr, T. Phys. Rev. A 1984, 30, 1960

(14) Ringland, J.; Issa, N.; Schell, M. Phys. Rev. A 1990, 41, 4223.

(15) Gaspard, P.; Nicolis, G. J. Stat. Phys. 1983, 31, 499. 

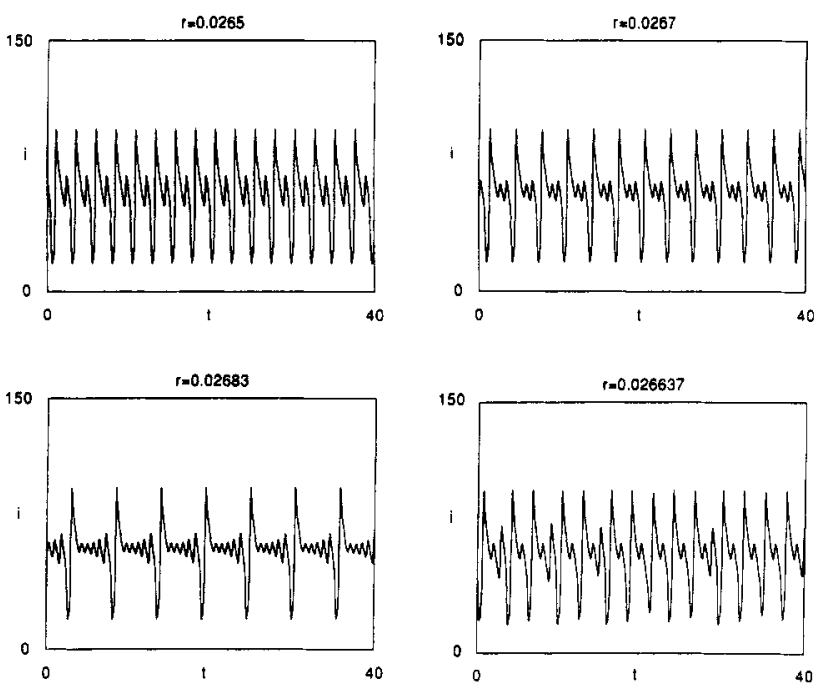

Figure 3. Typical oscillatory profiles of the flow defined by eqs 4-6 for higher values of the resistance $r$. Depicted are, for the indicated values of $r$, the MMO states $1^{1}, 1^{2}$, and $1^{5}$ and a chaotic state situated between the periodic states $1^{1}$ and $1^{2}$. All other parameter values as in Figure 1.

Relaxation Oscillation. From $r=0.01740$ to 0.02644 , the system shows the relaxation oscillation $1^{\circ}$ which is the dominant phenomenon observed in the In/SCN oscillator. ${ }^{6,10}$

MMO and Chaos. At higher $r$, we can again observe mixedmode oscillations, but now we may discern both chaotic and periodic states between two neighboring states, because contractivity during the reinjection is weaker here than in the MMO sequence at low $r$. The fixed point becomes a Shil'nikov saddle focus here as well, but again the complete homoclinic connection is not formed, the highest observed MMO being the $1^{5}$ state. Some of the typical time series observable in this $r$ domain are shown in Figure 3. Generally, periodic states turn into chaotic ones through subsequent subharmonic bifurcations, but for the chaotic states, however, we observed that both a tangent bifurcation and a cascade of period halvings ( $r$ increasing) can be the cause of their dislodgement. Again, this kind of behavior seems typical of a phase space flow that can be modeled with an iterative map that posseses two extrema. ${ }^{14}$ At $r=0.026843$, the prevailing MMO $1^{5}$ is suddenly destroyed discontinuously, giving a su-

(16) Gaspard, P; Kapral, R.; Nicolis, G. J. Stat. Phys. 1984, 35, 697. Glendinning, P.; Sparrow, C. J. Stat. Phys, 1984, 35, 645. bharmonic small-amplitude oscillation (period four). This state is rapidly period halved twice, and the oscillatory behavior is eventually killed when running into a Hopf bifurcation at $r=$ 0.02700 . Both the sequence and the oscillation profiles observed at these higher values of $r$ are once more very reminiscent of the experimental observations in the copper electrodissolution in phosphoric acid, though it should be added that these latter were obtained with a different kind of control parameter, namely, the rotation rate of the copper disk.'

High-Current Stationary State. After the last Hopf bifurcation, the system is in a stationary state where the electrode potential is low and the current is reaching its diffusion-limited regime.

The different dynamical behaviors and their bifurcations can be understood in terms of an S-shaped slow manifold corresponding to $\mathrm{de} / \mathrm{d} t=0$ in (4), which is caused by two largely different time scales in the system. ${ }^{17}$ The slow manifold arises because $r$ is small while the product $m r$ is of order 1 . The MMO at both ends of the oscillatory $r$ domain are due to the presence of a saddle focus at either one or the other fold of the slow manifold. This feature lies at the origin of the incompleteness of the homoclinic scenario in the present system and is reminiscent of the ducklike limit cycle analyzed by Baer and Erneux. ${ }^{18}$ The signature of this incomplete homoclinic scenario is the behavior (8) for the period of the MMO's near criticality.

To close this Letter, we may perhaps remark that the very satisfying agreement of our model predictions with the experiments is not the fortunate result of some artifact caused by our truncative approach. We observed exactly the same kind of behavior in a "many-box" description of the mass transport, closely resembling real Fick diffusion. Furthermore, we care to argue that the arguments that led us to introduce a third variable in our description of the diffusion are easily generalized to another kind of mass transport that is frequently encountered in electrochemistry: the coupling between diffusion and hydrodynamic convection at a rotating disk electrode. It is for this reason that our model may have wider applications, albeit perhaps in a little different appearance. In forthcoming work, ${ }^{19}$ we hope to elaborate in more detail on both the dynamical as well as the physicochemical properties of our model.

Acknowledgment. We thank Profs. G. Nicolis and J. H. Sluyters for suggestions and support of this research.

(17) Barkley, D. J. Chem. Phys. 1988, 89, 5547.

(18) Baer, S. M.; Erneux, T. SIAM J. Appl. Math. 1986, 46, 721.

(19) Koper, M.; Gaspard, P. Manuscript in preparation. 